\title{
Electrospray of a very viscous liquid in a dielectric liquid bath
}

\author{
F. J. Higuera \\ E. T. S. Ingenieros Aeronáuticos, UPM, Pza. Cardenal Cisneros 3, 28040 Madrid, Spain
}

\begin{abstract}
Numerical computations and order-of-magnitude estimates are used to analyze a jet of a very viscous liquid of finite electrical conductivity that is injected at a constant flow rate in an immiscible dielectric liquid under the action of an electric field. The conditions under which the injected liquid can form an elongated meniscus with a thin jet issuing from its apex (a cone-jet) are investigated by computing the flow, the electric field, and the transport of electric charge in the meniscus and a leading region of the jet. The boundaries of the domain of operation of the cone-jet mode are discussed. The current transfer region determining the electric current carried by the jet is analyzed taking into account the viscous drag of the dielectric liquid surrounding the jet. Conditions under which the electric current/flow rate characteristic follows a square root law or departs from it are discussed.
\end{abstract}

\section{INTRODUCTION}

Electrostatic dispersion is a technique used to generate emulsions of electrically conducting liquids in baths of immiscible dielectric liquids. It leads to drops with narrow distributions of sizes which can be controlled in the range from a few tens of nanometers to hundreds of micrometers, and therefore has potential applications to the synthesis of nanoparticles, the encapsulation of antibacterial or antifungal agents, and the manufacturing of drug-laden particles for targeted delivery, among others. The technique originated in the work of Barrero and coworkers [1,2], who showed that the cone-jet mode of an electrospray, which is often used to atomize conducting liquids in air or vacuum [3-8], can also be used in a bath of a dielectric liquid. These authors investigated fundamental properties of the cone-jet mode in a liquid bath, such as the current/flow rate and current/voltage characteristics, the scaling laws for the droplet size, and the enhanced whipping instability of the jets of these electrosprays compared with their counterparts in air. Gundabala and Fernández-Nieves [9] have carried out work along these lines for electrosprays embedded in microfluidic channels, and Alexander [10] investigated the pulsating modes of an electrospray in a liquid bath.

In this paper, numerical computations and order-of-magnitude estimates are used to fur- 


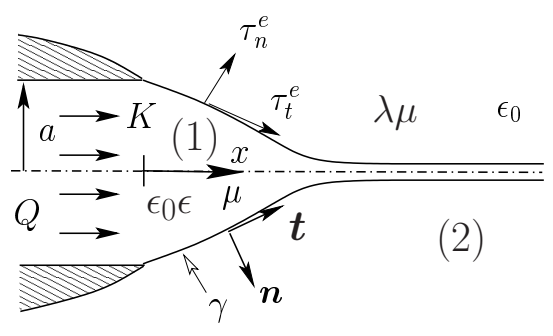

Figure 1: Sketch of the meniscus and the jet near the end of the capillary tube.

ther analyze the problem and propose scaling laws. Attention is focused on the stationary current transfer region of the electrospray's jet where convection of the electric charge accumulated at the interface between the two liquids begins to account for a fraction of the electric current of the spray. A square root current/flow rate characteristic is obtained in a certain range of flow rates, but the viscous drag due to the liquid bath may limit the length of the straight stationary jet and cause the electric current to fall below this square root law for large values of the flow rate.

\section{FORMULATION}

Figure 1 is a sketch of the system. The liquid to be dispersed (liquid 1) has viscosity $\mu$, electrical conductivity $\mu$ and permittivity $\epsilon_{0} \epsilon$. It is injected at a constant flow rate $Q$ through a metallic capillary tube of radius $a$ into a bath of an immiscible dielectric liquid (liquid 2) of viscosity $\lambda \mu$ and permittivity $\epsilon_{0}$ which is at rest far from the tube. Liquid 1 forms a meniscus at the end of the tube, which is subject to an electric field due to a high voltage applied between the capillary and a far electrode immersed in the bath. The electric field induces a conduction current in liquid 1 which accumulates electric charge at the interface between the two liquids. The field also causes electric stresses at the interface which must be balanced by pressure, viscous and surface tension stresses. The electric stresses elongate the meniscus in the direction of the field and, in certain conditions, lead to the formation of a thin jet that emerges from the tip of the meniscus and breaks into drops at some distance downstream. The effect of the inertia of the liquids is left out of the following analysis, which is a simplification appropriate for very viscous liquids in the meniscus and in an initial region of the jet; see, e.g., Ref. [11]. The flow in these regions is assumed to be stationary and axisymmetric.

In these condition, the governing equations are

$$
\begin{gathered}
\nabla^{2} \varphi_{i}=0 \\
\nabla \cdot \boldsymbol{v}_{i}=0, \quad 0=-\nabla p_{i}+\mu_{i} \nabla^{2} \boldsymbol{v}_{i}
\end{gathered}
$$


where the subscript $i$ denotes the variables in each liquid; $i=1,2$ with $\mu_{1}=\mu$ and $\mu_{2}=\lambda \mu$.

The boundary conditions at the interface between the two liquids are

$$
\begin{gathered}
\epsilon_{0}\left(E_{2 n}-\epsilon E_{1 n}\right)=\sigma, \quad E_{1 t}=E_{2 t} \\
\boldsymbol{v}_{1} \cdot \boldsymbol{\nabla} \sigma=K E_{1 n}+\sigma \boldsymbol{n} \cdot \boldsymbol{\nabla} \boldsymbol{v}_{1} \cdot \boldsymbol{n} \\
\boldsymbol{v}_{1} \cdot \boldsymbol{\nabla f}=0, \quad \boldsymbol{v}_{1}=\boldsymbol{v}_{2} \\
p_{2}-p_{1}+\boldsymbol{n} \cdot\left(\tau_{1}^{\prime}-\tau_{2}^{\prime}\right) \cdot \boldsymbol{n}+\gamma \boldsymbol{\nabla} \cdot \boldsymbol{n}=\tau_{n}^{e} \\
\boldsymbol{t} \cdot\left(\tau_{1}^{\prime}-\tau_{2}^{\prime}\right) \cdot \boldsymbol{n}=\tau_{t}^{e} .
\end{gathered}
$$

Equations (6) and (7) are balances of stresses normal and tangent to the interface. Here $p_{1}$ and $p_{2}$ are the pressures of the liquids, $\tau_{1}^{\prime}$ and $\tau_{2}^{\prime}$ are the viscous stress tensors, and $\gamma$ is the interfacial tension The electric stresses normal and tangent to the interface, in the right-hand sides of these equations, are (Landau and Lifshitz [12] and Saville [13])

$$
\tau_{n}^{e}=\frac{\epsilon_{0}}{2}\left(E_{2 n}^{2}-\epsilon E_{1 n}^{2}\right)+\frac{\epsilon_{0}}{2}(\epsilon-1) E_{1 t}^{2} \quad \text { and } \quad \tau_{t}^{e}=\sigma E_{1 t}
$$

in terms of the density of free surface charge $\sigma$ and the normal and tangent components of the electric field at the two sides of the interface $\left(E_{i n}=\boldsymbol{E}_{i} \cdot \boldsymbol{n}\right.$ and $E_{i t}=\boldsymbol{E}_{i} \cdot \boldsymbol{t}$, where $\boldsymbol{n}$ and $\boldsymbol{t}$ are unit vectors normal and tangent to the interface; see Fig. 1).

The electric field in liquid $i$ is $\boldsymbol{E}_{i}=-\boldsymbol{\nabla} \varphi_{i}$, where the electric potentials $\varphi_{i}$ satisfy the Laplace's equations (1), to be solved with the electrostatic boundary conditions (3) at the interface, the condition that the surface of the tube is an equipotential, and that the electric potential away from the tube tends to the potential of a needle, $\varphi_{\infty}=V^{*} \ln \left\{\left[\left(x^{2}+r^{2}\right)^{1 / 2}+x\right] / a\right\}$, where $V^{*}$ is proportional to the voltage applied between the electrodes and $x$ and $r$ are distances along the axis of the tube and normal to the axis.

The density of surface charge obeys the transport equation (4), which expresses the condition that the charge of a material element of the interface increases at the rate at which conduction in the inner liquid (liquid 1) accumulates charge at the interface.

The velocity and pressure of each liquid are given by the mass and momentum conservation equations (2), to be solved with the boundary conditions (5)-(7) at the interface and the conditions that the velocity of the outer liquid (liquid 2) is zero at the outer surface of the tube and at infinity and that the velocity of the inner liquid is $Q /\left(\pi a^{2}\right)$ at the outlet of the tube $(x=0)$. Conditions (5) mean that the interface, which is sought in the form $r=r_{s}(x)$ with $r_{s}(0)=a$, is a material surface.

The electrical and mechanical problems are coupled through the electric stresses at the interface.

The problem can be written in dimensionless variables using the radius of the tube $a$, the viscous-capillary velocity $v_{c}=\gamma / \mu$, and the field $E_{c}=\left(\gamma / \epsilon_{0} a\right)^{1 / 2}$ as scales of length, velocity 
and electric field. The solution depends on the five dimensionless parameters

$$
C a=\frac{\mu Q}{\gamma a^{2}}, \quad V=\frac{\epsilon_{0}^{1 / 2} V^{*}}{\gamma^{1 / 2} a^{1 / 2}}, \quad \epsilon, \quad \Lambda=\frac{\mu K a}{\epsilon_{0} \gamma}, \quad \lambda .
$$

\section{RESULTS AND DISCUSSION}

\section{Numerical results}

Figure 2 shows different elements of the solution for $V=1.8, \epsilon=20, \Lambda=10^{3}, \lambda=0.03$, and three values of the dimensionless flow rate $C a$, as functions of the distance $x$ to the tube. Figure 1(a) shows longitudinal sections of the meniscus and the jet. As can be seen, the size of the meniscus and the radius of the jet increase with the flow rate.

Figure 2(b) shows the contributions of conduction in the inner liquid ( $I_{b}=2 \pi K \int_{0}^{r_{s}} E_{1 x} r \mathrm{~d} r$, where $E_{1 x}$ is the axial field in the inner liquid; dashed curves) and convection of the surface charge $\left(I_{s}=2 \pi \sigma v r_{s}\right.$; solid curves) to the electric current. The sum $I=I_{b}+I_{s}$ is a constant, equal to the current carried by the jet. The conduction current $I_{b}$ dominates in the meniscus and the convection current $I_{s}$ dominates in the jet where, however, the conduction current does not seem to go to zero. The cross-over point where the two contributions to the current are equal to each other is well into the jet and shifts streamwise when the flow rate increases.

Figure 2(c) shows the electric stress tangent to the surface (dashed curves) and the difference between the normal electric stress and the surface tension stress (solid curves; here $\mathcal{C}$ is twice the mean curvature of the interface). The tangent electric stress drives the flow in the jet. However, this force is small in the meniscus, where the interface is nearly equipotential and the tangent electric field is small, and rises to a maximum in the current transfer region around the cross-over point where the two contributions to the current are of the same order.

The momentum equation for the inner liquid in $(2)(i=1)$ can be integrated across the jet, where the flow is quasi-unidirectional, to give the balance of forces (see, e.g., GañánCalvo et al. [14] and Feng [15])

$$
\frac{\partial}{\partial x}\left(3 \pi r_{s}^{2} \mu \frac{\partial v}{\partial x}\right)+\pi r_{s}^{2} \frac{\partial}{\partial x}\left(\tau_{n}^{e}-\frac{\gamma}{r_{s}}+\tau_{n}^{o}\right)+2 \pi r_{s}\left(\tau_{t}^{e}+\tau_{t}^{o}\right)=0
$$

where the terms on the left-hand side are the axial forces per unit length of the jet due to the effective axial viscous stress in the inner liquid; to the axial gradient of the pressure variation induced by the normal electric stress, the surface tension stress and the stress of the outer liquid normal to the interface $\left(\tau_{n}^{o}=-p_{2}+\boldsymbol{n} \cdot \tau_{2}^{\prime} \cdot \boldsymbol{n}\right)$; and the forces due to the electric shear stress $\tau_{t}^{e}$ and the viscous shear stress of the outer liquid $\left(\tau_{t}^{o}=\boldsymbol{t} \cdot \tau_{2}^{\prime} \cdot \boldsymbol{n}\right)$. 

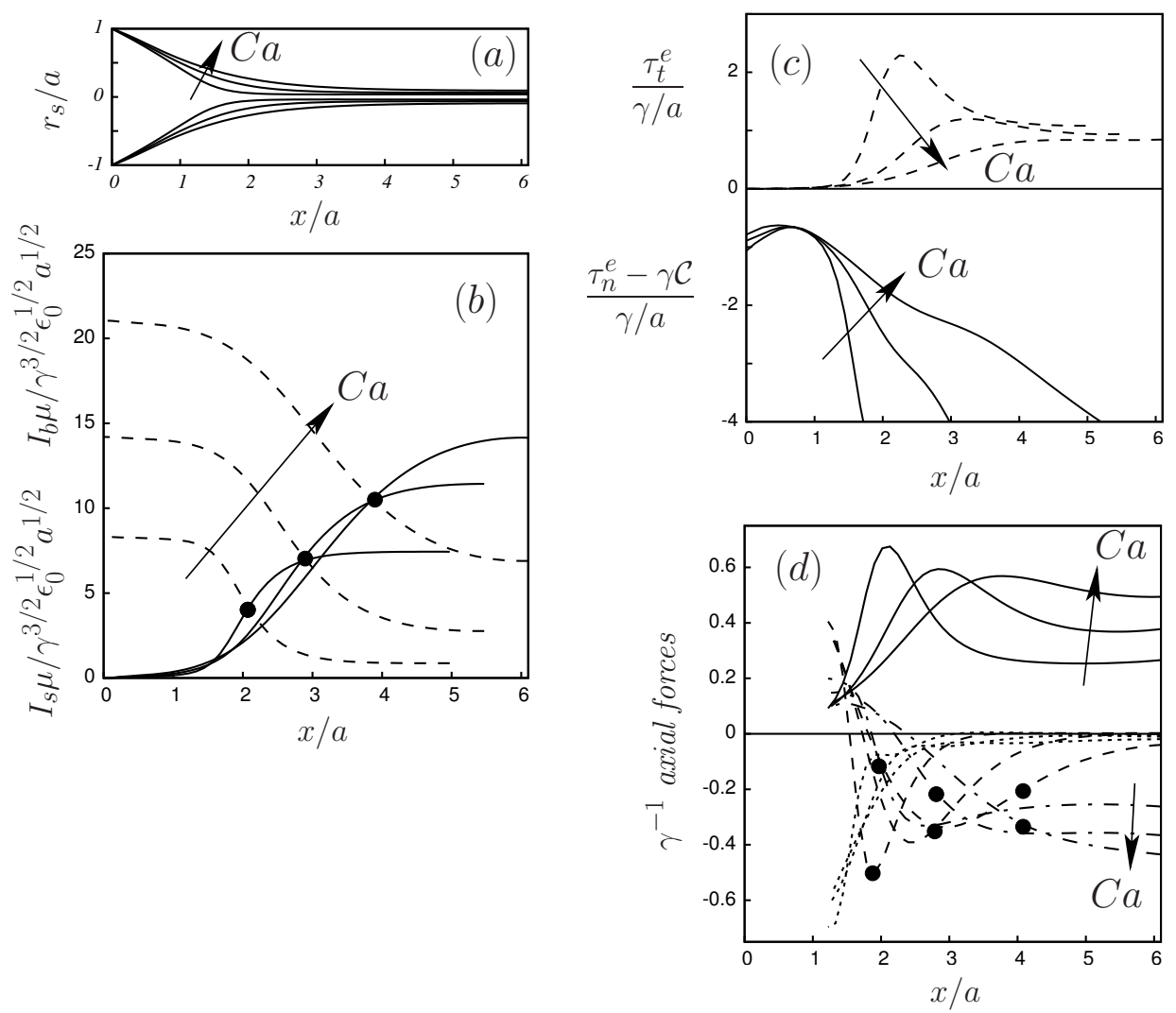

Figure 2: (a) Longitudinal sections of the interface. (b) Convection (solid) and conduction (dashed) contributions to the electric current nondimensionalized with $\epsilon_{0} E_{c} v_{c} a$ [see definitions above Eq. (9)]. (c) Stresses normal $\left(\tau_{n}^{e}-\gamma \mathcal{C}\right.$, solid) and tangent $\left(\tau_{t}^{e}\right.$, dashed) to the interface nondimensionalized with $\gamma / a$. (d) Axial forces acting on the jet per unit streamwise length nondimensionalized with $\gamma$. Electric force $2 \pi r_{s} \tau_{t}^{e}$ in the last term of (10) (solid); force due to the axial viscous stress in the inner liquid, $\partial\left(3 \pi r_{s}^{2} \mu \partial v / \partial x\right) / \partial x$ in (10) (dashed); force due to the normal electric stress and the surface tension, $\pi r_{s}^{2} \partial\left(\tau_{n}^{e}-\gamma / r_{s}\right) / \partial x$ in (10) (dotted); and force due to the viscous shear stress of the outer liquid, $2 \pi r_{s} \tau_{t}^{o}$ in the last term of (10) (chain). Values of the parameters are $C a=0.0256,0.0858$ and 0.1977 (increasing as indicated by the arrows), $V=1.8, \epsilon=20, \Lambda=10^{3}$ and $\lambda=0.03$. The black circles in $(b)$ and $(d)$ mark the crossover point at which the two contributions to the current are equal to each other. 
These forces are evaluated from the full numerical solution and shown in Fig. 2(d). In the current transfer region, the force due to the electric shear stress (solid curves) is balanced mainly by the forces due to the axial viscous stress of the inner liquid (dashed curves) and the viscous shear stress of the outer liquid (chain curves). The values of these two forces at the current cross-over point are marked by black circles. As can be seen, the force due to the viscosity of the inner liquid dominates at small flow rates and the drag of the outer liquid becomes more important when the flow rate increases. The first of these forces tends to zero far downstream, leaving a balance of electrical and viscous shear stresses in the far jet.
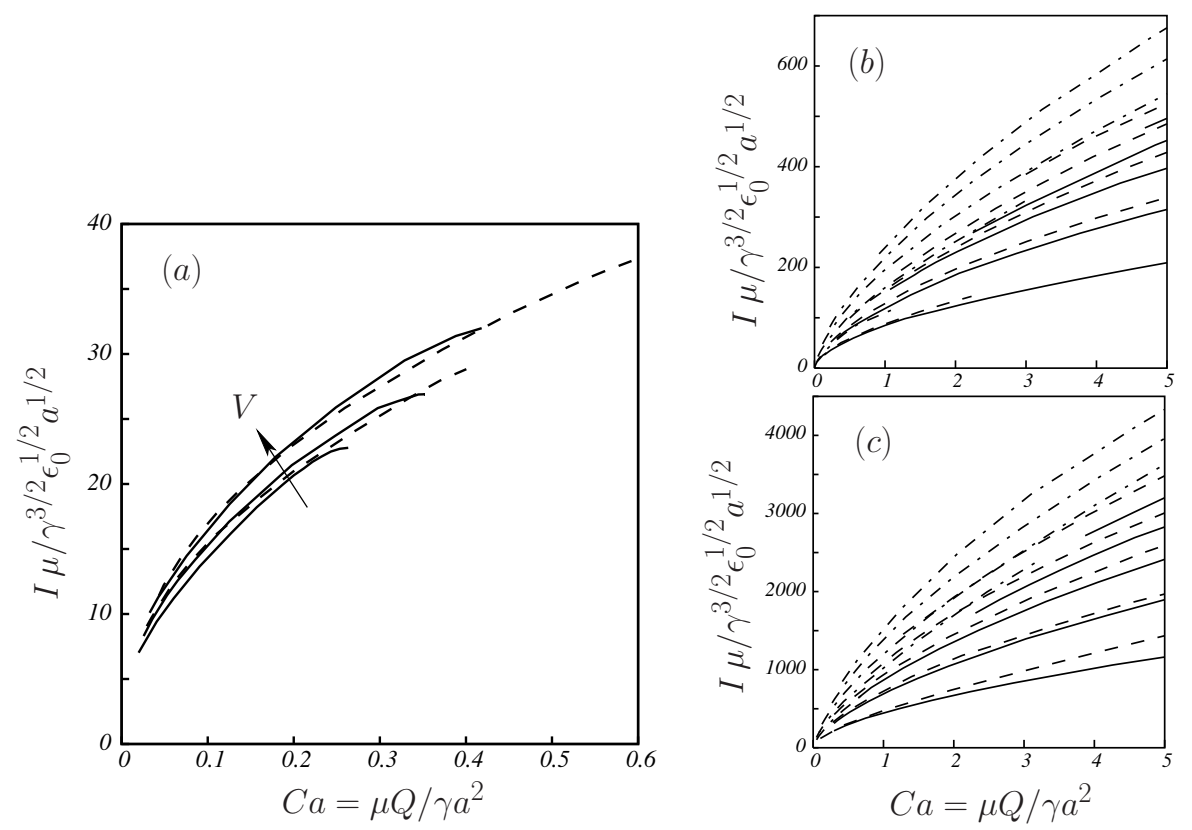

Figure 3: (a) Current/flow rate characteristic for $\lambda=0.01, V=1.6$ and 1.8 (dashed curves), and for $\lambda=0.03, V=1.6,1.8$ and 2 (solid curves). Values of other parameters are $\epsilon=20$ and $\Lambda=10^{3}$. ( $b$ and $c$ ) Current/flow rate characteristic for two parallel plate electrodes. Here $\lambda=0$ (solid), 0.01 (dashed) and 0.1 (chain), $E_{\infty}=1,2,3,4,5$, increasing from bottom to top, $\epsilon=20$, and $\Lambda=10^{3}(b)$ and $2 \times 10^{4}(c)$.

The electric current carried by the jet is shown in Fig. 3(a) as a function of the dimensionless flow rate $\mathrm{Ca}$ for different values of the other dimensionless parameters. The current increases nearly as the square root of the flow rate in a certain range of this variable. The current also increases with the applied voltage $V$ and with the ratio of outer-to-inner liquid viscosity $\lambda$.

There is no stationary solution below a certain minimum flow rate, near which the meniscus is conical and the jet is very thin. There is also a maximum flow rate near which the current falls below the square root law and seems to level to a constant value before the com- 
putations cease to converge to a stationary solution. The departure from the square root law is more pronounced and occurs earlier when the viscosity of the outer liquid increases. The volume of the meniscus and the radius of the jet increase with the flow rate, and the meniscus does not resemble a Taylor cone at the maximum flow rate.

Figures 3(b,c) show the electric current as a function of the flow rate for a parallel plate electrode configuration, for which the electric field tends to a constant ( $E_{\infty}$ in dimensionless variables) far from the meniscus. The results for this case are similar to those of Fig. 3(a) for a needle-plate electrode configuration, but the current increases with a different power of the flow rate, which is about $2 / 3$.

\section{Order-of-magnitude estimates}

The following three conditions are satisfied in the current transfer region of the jet.

- The conduction and convection contributions to the current are of the same order. This condition, which defines the current transfer region, can be written in the form

$$
K E_{T} r_{s}^{2} \sim \sigma v r_{s} \quad \text { with } E_{T} \sim\left(\frac{\gamma}{\epsilon_{0} x}\right)^{1 / 2} \text { and } v \sim \frac{Q}{r_{s}^{2}} .
$$

Here $E_{T}$ is the electric field acting on the jet, which is the Taylor field due to the conical meniscus. The velocity of the liquid can be written in terms of the flow rate and the local radius of the jet.

- The axial electric field induced by the charge at the surface of the jet, which acts as a line distribution of charge, is of the order of the Taylor field $E_{T}$ and partially balances it at the surface. In orders of magnitude and leaving out a logarithmic factor [16], this condition reads

$$
E_{2 n} \frac{r_{s}}{x} \sim E_{T} \quad \text { with } \quad \epsilon_{0} E_{2 n} \sim \sigma
$$

This screening condition is a consequence of the conductivity of the inner liquid, which tends to accumulate electric charge at the interface until this charge screens the inner liquid from the outer field and conduction ceases. However, the screening cannot be complete over the whole jet when the liquid is in motion, because the flow removes surface charge from any given region of the interface and this charge must be continuously replaced by new charge carried to the interface by conduction in the inner liquid. This, in turn, requires an electric field in the inner liquid, and this field increases with the length of the region to be screened. Since the inner field cannot be larger than the outer field, the condition that the two fields be of the same order determines the length of the region that can be partially screened, which coincides with the current transfer region where the outer field $E_{T}$ first enters the inner liquid. 
- The third condition to be satisfied is a balance of forces. The force driving the flow in the current transfer region, due to the electric shear stress at the interface, is of order $r_{s} \sigma E_{T}$. In the absence of inertia, this force must be balanced by $(a)$ the force due to the axial viscous stress in the inner liquid (first term of (10), which is of order $r_{s}^{2} \mu v / x^{2}$ ), or $(b)$ the force due to the viscous shear stress of the outer liquid (last term of (10), which is of order $r_{s} \lambda \mu v / r_{s}$, where it is assumed that the thickness of layer of outer liquid set in motion by the electric force is of the order of the radius of the jet or larger than it; see, e.g., Ref. [11] for details).

The relevant balance of forces, together with conditions (11) and (12), determines the characteristic length of the current transfer region and the characteristic radius of the jet in this region as

$$
x \sim \frac{\mu^{1 / 2} Q^{1 / 2}}{\gamma^{1 / 2}}, \quad r_{s} \sim \frac{\epsilon_{0}^{1 / 4} \mu^{1 / 8} Q^{3 / 8}}{K^{1 / 4} \gamma^{1 / 8}}
$$

in case $(a)$ and

$$
x \sim \frac{\lambda^{2} \mu^{2} K Q}{\epsilon_{0} \gamma^{2}}, \quad r_{s} \sim \frac{\lambda^{1 / 2} \mu^{1 / 2} Q^{1 / 2}}{\gamma^{1 / 2}}
$$

in case $(b)$.

The characteristic current carried by the jet follows the square root law

$$
I \sim(\gamma K Q)^{1 / 2}
$$

in the two cases.

Which of the two possibilities (13) or (14) is realized for a given set of values of the parameters can be determined by comparing the estimates of the forces in (10). Thus,

$$
\frac{2 \pi r_{s} \tau_{t}^{o}}{\partial\left(3 \pi r_{s}^{2} \mu \partial v / \partial x\right) / \partial x} \sim \Pi=\frac{\lambda \mu^{3 / 4} K^{1 / 2} Q^{1 / 4}}{\epsilon_{0}^{1 / 2} \gamma^{3 / 4}}
$$

when (13) is used. Therefore the drag of the outer liquid is the dominant force to be balanced by the electric shear in the current transfer region if $\Pi$ is large compared to unity, and this drag is negligible in the current transfer region if $\Pi$ is small compared to unity.

The condition used in (12), that the density of surface charge in the current transfer region is of the order of the equilibrium charge density that would electrically screen the inner liquid, requires that the time available for conduction to charge the interface, which is of the order of the residence time $t_{r}=x / v$, be large compared to the electric relaxation time of the inner liquid $t_{e}=\epsilon_{0} \epsilon / K$. The ratio $t_{r} / t_{e}$ is an increasing function of the flow rate in the two possible cases. The condition that this ratio be of order unity determines 
the order of the flow rate at which charge relaxation effects come into play in the current transfer region as

$$
Q_{\min }^{(a)} \sim \frac{\epsilon^{4} \epsilon_{0}^{2} \gamma^{3}}{\mu^{3} K^{2}} \quad \text { or } \quad Q_{\min }^{(b)} \sim \frac{\epsilon \epsilon_{0}^{2} \gamma^{3}}{\lambda^{3} \mu^{3} K^{2}}
$$

These estimates give also a lower bound of the range of flow rates where the square root law for the current (15) can be realized. Which of the two estimates (17) is relevant depends on the value of the product $\lambda \epsilon$. If this product is small, then the drag of the outer liquid becomes small compared to the force due to the axial viscous stress in the inner liquid before charge relaxation effects appear, which happens when the flow rate decreases to values of order $Q_{\text {min }}^{(a)}$. On the other hand, the drag of the outer liquid dominates and charge relaxation effects appear for flow rates of order $Q_{\text {min }}^{(b)}$ if the product $\lambda \epsilon$ is large.

Convection of the surface charge makes an important contribution to the total electric current downstream of the current transfer region. This determines the order of the density of surface charge in the far jet as $\sigma \sim I r_{s} / Q$, and the electric shear stress as $\tau_{t}^{e}=\sigma E_{\infty}(x)$, where $E_{\infty}(x)$ is the electric field acting on the jet in this far region. In addition, the equilibrium of the driving electrical force and the viscous force of the inner liquid or the drag of the outer liquid determines the evolution of the radius of the jet as

$$
\frac{r_{s}^{2} \mu v}{x^{2}} \sim r_{s} \tau_{t}^{e} \Rightarrow r_{s} \sim\left(\frac{\mu Q^{2}}{I x^{2} E_{\infty}}\right)^{1 / 2}
$$

or

$$
\frac{\lambda \mu v}{r_{s}} \sim \tau_{t}^{e} \Rightarrow r_{s} \sim\left(\frac{\lambda^{2} \mu^{2} Q^{2}}{I E_{\infty}}\right)^{1 / 4}
$$

The second possibility, corresponding to the equilibrium of electric shear stress and viscous shear stress of the outer liquid, should be always realized sufficiently far downstream for any $\lambda>0$. However, the jet may break into drops or undergo other instability before reaching the final state (19). If this state is attained and the field $E_{\infty}$ acting on the far jet is nearly uniform, which is probably the case in the experiments of Riboux et al. [2] carried out with a needle-plate electrode configuration, then the radius of the jet and the conduction current are predicted to reach constant values independent of streamwise distance. This result agrees with the experimental observations in Ref. [2].

The first result (13) predicts that the length of the current transfer region increases linearly with the flow rate in the case when the drag of the outer liquid is large compared to the force due to the axial viscous stress of the inner liquid in the current transfer region. The length $x$ becomes then of the order of the radius of the capillary $a$ when the flow rate becomes of the order of

$$
Q_{\max }=\frac{\epsilon_{0} \gamma^{2} a}{\lambda^{2} \mu^{2} K}
$$


The estimates leading to (13) should be modified for $Q \gg Q_{\max }$ because the transfer of current to the surface of the jet would occur in a region where the electric field acting on the jet is not the field of a Taylor cone but decreases as the inverse of the distance to the tube. This changes the electric force on the jet, and the balance of this force and the drag of the outer liquid gives a radius of the jet than increases with streamwise distance upstream of the current transfer region, where the electric shear stress is still small and the force driving the flow is due to the gradient of the depression induced in the inner liquid by the electric stress normal to its surface [second term of (10)]. But this result is meaningless because the latter force points toward the meniscus when $r_{s}$ increases with $x$. This negative result means that the straight stationary jet cannot extend to distances downstream of the tube much larger than its radius $a$. The jet is expected to break up or undergo whipping almost immediately upon being injected when $Q$ is of the order of $Q_{\max }$ or larger. The current that can be transferred to the surface in the limited region where the jet is straight and stationary can be easily estimated and turns out to be independent of the flow rate, which is in qualitative agreement with experiments [2, 9].

The numerical results show that the ratio of the force due to the electrically induced depression to the drag of the outer liquid decreases at the current cross-over point when the flow rate increases. This means that the equilibrium of forces in the current transfer region depends more and more on the electric shear stress. Apparently this equilibrium ceases to be possible when the rise of the electric shear stress is postponed, by increasing the flow rate, beyond the region where the electric field is that of a Taylor cone.

\section{SUMMARY}

The flow, the electric field and the surface charge of a stationary electrified jet of a very viscous liquid injected into a quiescent immiscible dielectric liquid have been computed numerically.

Qualitative estimates have been worked out for the current transfer region of the jet under different conditions of the flow.

A square root current/flow rate characteristic is found in a certain range of flow rates.

Charge relaxation effects come into play in the current transfer region when the flow rate approaches a certain minimum which depends on the properties of the liquids.

A maximum flow rate is found above which no long stationary jet can exist.

\section{ACKNOWLEDGMENTS}

This work was supported by the Spanish Ministerio de Educación y Ciencia through 
Project No. DPI2007-66659-C03-02.

\section{References}

[1] A. Barrero, J. M. López-Herrera, A. Boucard, I. G. Loscertales and M. Márquez, J. Colloid Interface Sci. 272, 104 (2004).

[2] G. Riboux, A. G. Marín, I. G. Loscertales and A. Barrero, J. Fluid Mech. Submitted (2010). Also Procs. 1st European Conf. on Microfluidics, Bologna, December 10-12 (2008).

[3] J. Zeleny, Proc. Camb. Phil. Soc. 18, 1 (1015).

[4] G. I. Taylor, Proc. R. Soc. Lond. A 280, 383 (1964).

[5] D. P. H. Smith, IEEE Trans. Ind. Appl. IA22, 527 (1986).

[6] M. Cloupeau and B. Prunet-Foch, J. Electrost. 22, 135 (1989).

[7] J. Fernández de la Mora and I. G. Loscertales, J. Fluid Mech. 260, 155 (1994).

[8] J. Fernández de la Mora, Ann. Rev. Fluid Mech. 39, 217 (2007).

[9] V. R. Gundabala and A. Fernández-Nieves, Phys. Rev. Lett. Submitted (2010).

[10] M. S. Alexander, Appl. Phys. Lett. 92, 144102 (2008).

[11] F. J. Higuera, J. Fluid Mech. 648, 35 (2010).

[12] L. D. Landau and E. M. Lifshitz, Electrodynamics of Continuous Media (Pergamon, Oxford, 1960).

[13] D. A. Saville, Ann. Rev. Fluid Mech. 29, 27 (1997).

[14] A. M. Gañán-Calvo, J. Dávila and A. Barrero, J. Aerosol Sci. 28, 249 (1997).

[15] J. J. Feng, Phys. Fluids 14, 3912 (2002).

[16] E. J. Hinch, Perturbation Methods (Cambridge University Press, Cambridge, 1991). 